Research Paper

\title{
Dexmedetomidine Inhibits Phenylephrine-induced Contractions via Alpha-1 Adrenoceptor Blockade and Nitric Oxide Release in Isolated Rat Aortae
}

\author{
Hyo-Jin Byon ${ }^{*}$, Seong-Ho Ok²*, Soo Hee Lee², Sebin Kang³, Youngil Cho³, Jeong Yeol Han ${ }^{4}$, Ju-Tae Sohn²,5凶 \\ 1. Department of Anesthesiology and Pain Medicine, Anesthesia and Pain Research Institute, Yonsei University College of Medicine, Seoul, Korea; \\ 2. Department of Anesthesiology and Pain Medicine, Gyeongsang National University School of Medicine, Gyeongsang National University Hospital, Jinju, \\ 52727, Republic of Korea; \\ 3. Department of Anesthesiology and Pain Medicine, Gyeongsang National University Hospital, Jinju, 52727, Republic of Korea; \\ 4. Department of Anesthesiology and Pain Medicine, Gyeongsang National University Changwon Hospital, Changwon, 51427, Republic of Korea; \\ 5. Institute of Health Sciences, Gyeongsang National University, Jinju-si, Republic of Korea. \\ *These authors equally contributed to this study as co-first authors.
}

$\square$ Corresponding author: Ju-Tae Sohn, Department of Anesthesiology and Pain Medicine, Gyeongsang National University Hospital, Jinju, 52727, Republic of Korea; Tel.: +82-55-750-8586; Fax: +82-55-750-8142; E-mail: jtsohn@nongae.gsnu.ac.kr.

(c) Ivyspring International Publisher. This is an open access article distributed under the terms of the Creative Commons Attribution (CC BY-NC) license (https://creativecommons.org/licenses/by-nc/4.0/). See http://ivyspring.com/terms for full terms and conditions

Received: 2016.09.03; Accepted: 2016.12.21; Published: 2017.02.07

\begin{abstract}
The goal of this in vitro study was to examine the effect of the alpha-2 adrenoceptor agonist dexmedetomidine on phenylephrine (alpha-1 adrenoceptor agonist)-induced contraction in isolated rat aortae and to elucidate the associated cellular mechanisms, with a particular focus on alpha-1 adrenoceptor antagonism. Dexmedetomidine dose-response curves were generated in isolated endothelium-intact and endothelium-denuded rat aortae precontracted with phenylephrine or 5-hydroxytryptamine. Endothelium-denuded aortic rings were pretreated with either dexmedetomidine or the reversible alpha-1 adrenoceptor antagonist phentolamine, followed by post-treatment with the irreversible alpha-1 adrenoceptor blocker phenoxybenzamine. Control rings were treated with phenoxybenzamine alone. All rings were repeatedly washed with Krebs solution to remove all pretreatment drugs, including phenoxybenzamine, phentolamine and dexmedetomidine. Phenylephrine dose-response curves were then generated. The effect of rauwolscine on the dexmedetomidine-mediated change in phenylephrine-induced endothelial nitric oxide synthase (eNOS) phosphorylation in human umbilical vein endothelial cells was examined using western blotting. The magnitude of the dexmedetomidine-mediated inhibition of phenylephrine-induced contraction was higher in endothelium-intact aortae than in endothelium-denuded aortae or endothelium-intact aortae treated with $\mathrm{N}^{\omega}$-nitro-L-arginine methyl ester. However, dexmedetomidine did not significantly alter 5-hydroxytryptamine-induced contraction. In further experiments, prazosin attenuated dexmedetomidine-induced contraction. Additionally, pretreatment with either dexmedetomidine plus phenoxybenzamine or phentolamine plus phenoxybenzamine produced greater phenylephrine-induced contraction than phenoxybenzamine alone, suggesting that dexmedetomidine protects aortae from the alpha-l adrenoceptor blockade induced by phenoxybenzamine. Rauwolscine attenuated the dexmedetomidine-mediated enhancement of phenylephrine-induced eNOS phosphorylation. Taken together, these results suggest that dexmedetomidine attenuates phenylephrine-induced contractions via alpha-1 adrenoceptor blockade and endothelial nitric oxide release in the isolated rat aorta.
\end{abstract}

Key words: dexmedetomidine, phenylephrine, alpha-2 adrenoceptor agonist, alpha-1 adrenoceptor, contraction, aorta, phenoxybenzamine, phentolamine, nitric oxide. 


\section{Introduction}

The highly selective alpha- 2 adrenoceptor agonist dexmedetomidine is widely used for sedation in perioperative periods [1,2]. Intravenous administration of dexmedetomidine produces transient hypertension followed by hypotension, due to alpha-2 adrenoceptor-mediated vasoconstriction and a central sympatholytic effect, respectively [1-3]. The hypotension caused by dexmedetomidine infusion during various procedures requires a large amount of phenylephrine [4-6]. Furthermore, dexmedetomidine attenuates the contraction induced by the alpha- 1 adrenoceptor agonist phenylephrine in isolated vessels $[7,8]$. In addition, it has been reported that dexmedetomidine shows low affinity for the alpha-1 adrenoceptor $[9,10]$. These previous reports suggest that high doses of dexmedetomidine induce competitive inhibition at the alpha- 1 adrenoceptor via the intrinsic low affinity of dexmedetomidine for the alpha-1 adrenoceptor [7-10]. The vasoconstriction induced by dexmedetomidine is attenuated by endothelial nitric oxide in isolated endothelium-intact rat aortae [11]. However, the detailed cellular mechanism responsible for the dexmedetomidinemediated inhibition of alpha-1 adrenoceptor-induced contraction remains unknown. Therefore, the goal of this in vitro study was to examine the effect of dexmedetomidine on phenylephrine-induced contraction in isolated rat aorta and to elucidate the associated cellular mechanism, with a particular focus on the antagonistic effects on the alpha-1 adrenoceptor. We tested the hypothesis that dexmedetomidine attenuates phenylephrine-induced contraction via both inhibition of the alpha-1 adrenoceptor and activation of endothelial nitric oxide release.

\section{Materials and Methods}

All experimental procedures and protocols were approved by the Institutional Animal Care and Use Committee of Gyeongsang National University and were performed in accordance with the Guide for the Care and Use of Laboratory Animals as described previously [12].

\section{Preparation of aortic rings for tension measurements}

Aortic rings were prepared for tension measurements as described previously [12-14]. Male Sprague-Dawley rats weighing 250-300 g were anesthetized via intramuscular injection of Zoletil 50 (15 mg/kg, Virbac Laboratories, Carros, France). The descending thoracic aorta was then dissected free. The surrounding connective tissue and fat were removed under a microscope, and the vessel was bathed in Krebs solution with the following composition (mM): $118 \mathrm{NaCl}, 4.7 \mathrm{KCl}, 1.2 \mathrm{MgSO}_{4}, 1.2 \mathrm{KH}_{2} \mathrm{PO}_{4}, 2.4 \mathrm{CaCl}_{2}$, $25 \mathrm{NaHCO}_{3}$, and 11 glucose. The aorta was then cut into 2.5-mm rings, which were suspended on Grass isometric transducers (FT-03, Grass Instrument, Quincy, MA, USA) under 3.0-g resting tension in a $10-\mathrm{mL}$ Krebs bath at $37^{\circ} \mathrm{C}$ and continuously aerated with $95 \% \mathrm{O}_{2}$ and $5 \% \mathrm{CO}_{2}$ to maintain $\mathrm{pH}$ values within 7.35-7.45. The rings were equilibrated at a resting tension of $3.0 \mathrm{~g}$ for $120 \mathrm{~min}$, during which the bathing solution was changed every $40 \mathrm{~min}$. Care was taken not to damage the endothelium. In some aortic rings, the endothelium was intentionally removed by inserting a 25-gauge needle tip into the lumen of the ring and gently rolling the ring for a few seconds. Once the phenylephrine $\left(10^{-7} \mathrm{M}\right)$-induced contraction had stabilized, acetylcholine $\left(10^{-5} \mathrm{M}\right)$ was added to assess endothelial integrity. Endothelial integrity was confirmed by the observation of more than $75 \%$ relaxation induced by acetylcholine. Once the phenylephrine $\left(10^{-8} \quad \mathrm{M}\right)$-induced contraction was sustained in the endothelium-denuded rat aorta, acetylcholine was added to assess endothelial denudation; aortic rings showing lees than 20\% relaxation induced by acetylcholine were regarded as endothelium-denuded aortic rings in the current experiment. The rings were then rinsed with fresh Krebs solution to restore the resting tension. Next, the contraction induced by isotonic $60 \mathrm{mM} \mathrm{KCl}$ was measured, and the magnitude of isotonic $60 \mathrm{mM}$ $\mathrm{KCl}$-induced contraction was used as the reference value $(100 \%)$ for concentration-response curves induced by phenylephrine [13]. After washing out the $\mathrm{KCl}$ from the organ bath with fresh Krebs solution and reaching the baseline resting tension, the main experiments were performed as described in the experimental protocols.

\section{Experimental protocols}

We investigated the dexmedetomidine concentration $\left(10^{-9}\right.$ to $\left.10^{-6} \mathrm{M}\right)$-response curves in isolated endothelium-intact aortae that were either precontracted with phenylephrine $\left(10^{-6} \mathrm{M}\right)$ or pretreated with the nitric oxide synthase inhibitor $\mathrm{N}^{\omega}{ }^{\omega}$-nitro-L-arginine methyl ester (L-NAME: $10^{-4} \mathrm{M}$ ) and precontracted with phenylephrine $\left(10^{-6} \mathrm{M}\right)$ as well as endothelium-denuded aortae pretreated with L-NAME $\left(10^{-4} \mathrm{M}\right)$ and precontracted with phenylephrine $\left(10^{-7} \mathrm{M}\right)$. We regarded isolated rat aortae showing less than $20 \%$ acetylcholine-induced relaxation as endothelium-denuded aortae in the current experiment, and endothelial nitric oxide attenuates dexmedetomidine-induced contraction [11]. As the residual endothelium of the endothelium- 
denuded aorta may affect dexmedetomidine concentration-response curves, endotheliumdenuded aortae were pretreated with $10^{-4} \mathrm{M}$ L-NAME for $15 \mathrm{~min}$ before the addition of phenylephrine. After phenylephrine had produced a stable and sustained contraction in the isolated rat aorta, dexmedetomidine was cumulatively added to generate dexmedetomidine concentration-response curves.

An alpha-1 adrenoceptor protection experiment was performed to determine whether the dexmedetomidine-mediated inhibition of vasoconstriction induced by the alpha- 1 adrenoceptor agonist phenylephrine involves alpha-1 adrenoceptor blockade $[15,16]$. Endothelium-denuded rat aortae were pretreated with either dexmedetomidine $(3 \times$ $10^{-7}$, or $10^{-6} \mathrm{M}$ ) or the reversible alpha- 1 adrenoceptor antagonist phentolamine $\left(10^{-6} \mathrm{M}\right)$ for $20 \mathrm{~min}$, followed by post-treatment with the irreversible alpha- 1 adrenoceptor antagonist phenoxybenzamine $\left(5 \times 10^{-8}\right.$ M) for another $20 \mathrm{~min}$. Control rings were treated with phenoxybenzamine $\left(5 \times 10^{-8} \mathrm{M}\right)$ alone for $20 \mathrm{~min}$. All rings were repeatedly washed with fresh Krebs solution every $5 \mathrm{~min}$ for $60 \mathrm{~min}$ to remove all of the pretreatment drugs, including phenoxybenzamine, dexmedetomidine and phentolamine, and to restore the baseline resting tension. Then, phenylephrine concentration $\left(10^{-9}\right.$ to $\left.10^{-5} \mathrm{M}\right)$-response curves were generated in the endothelium-denuded washed aorta pretreated with L-NAME $\left(10^{-4} \mathrm{M}\right)$.

We investigated the effect of the cumulative addition of dexmedetomidine on the contraction induced by phenylephrine $\left(1^{-7} \quad \mathrm{M}\right)$ or 5-hydroxytryptamine $\left(3 \times 10^{-6} \mathrm{M}\right)$ in an isolated L-NAME $\left(10^{-4} \mathrm{M}\right)$-pretreated endothelium-denuded rat aorta to determine whether the inhibitory effect of dexmedetomidine on phenylephrine-induced contraction is specifically associated with inhibitory effects on the alpha-1 adrenoceptor. After phenylephrine or 5-hydroxytryptamine had produced a sustained and stable contraction in the endothelium-denuded rat aorta pretreated with L-NAME $\left(10^{-4} \mathrm{M}\right)$, dexmedetomidine $\left(10^{-9}\right.$ to $\left.10^{-6} \mathrm{M}\right)$ was cumulatively added to generate dexmedetomidine concentration-response curves. In addition, we generated prazosin concentration $\left(10^{-10}\right.$ to $3 \times 10^{-9}$ $\mathrm{M})$-response curves in endothelium-denuded aortae that were pretreated with L-NAME $\left(10^{-4} \mathrm{M}\right)$ and precontracted with dexmedetomidine $\left(10^{-6} \mathrm{M}\right)$.

\section{Cell culture}

Human umbilical vein endothelial cells (HUVECs; EA.hy926 cells, American Type Culture Collection, Manassas, VA, USA) were grown in Dulbecco's modified Eagle's medium (DMEM) supplemented with $10 \%$ fetal bovine serum (FBS), 2 $\mathrm{mmol} / \mathrm{L}$ L-glutamine, $100 \mathrm{IU} / \mathrm{mL}$ penicillin, and 10 $\mu \mathrm{g} / \mathrm{mL}$ streptomycin as described previously [12]. The cells were cultured in 100-mm dishes and grown in a humidified $5 \% \mathrm{CO}_{2}$ incubator. HUVECs were plated at a density of $10^{7}$ cells per $100-\mathrm{mm}$ dish. The cells were used between passage number 7 and passage number 10 .

\section{Western blot analysis}

Western blot analysis was performed as previously described [12]. Briefly, cells were lysed in PRO-PREP protein extract solution to isolate the total cell extract. After the extract was centrifuged at 13,000 rpm for $20 \mathrm{~min}$ at $4^{\circ} \mathrm{C}$, the protein concentration was determined via the Bradford method. Samples containing $30 \mu \mathrm{g}$ of protein were subjected to $10 \%$ sodium dodecyl sulfate (SDS)-polyacrylamide gel electrophoresis. The separated proteins were then transferred to polyvinylidene difluoride membranes using the SD Semi-dry Transfer Cell ${ }^{\circledR}$ system (Bio-Rad, Hercules, CA, USA). These membranes were incubated with primary antibodies (anti-endothelial nitric oxide synthase [eNOS] and anti-phospho-eNOS at Ser1177 antibodies; Cell Signaling Technology, Beverly, MA, USA) at a 1:500 dilution $(4 \mu \mathrm{g} / \mathrm{mL})$ in $5 \%$ skim milk in Tris-buffered saline with Tween 20 overnight at $4^{\circ} \mathrm{C}$, and bound antibody was detected with horseradish peroxidase-conjugated anti-rabbit IgG. The membranes were washed and then developed using the Luminol Reagent system (Animal Genetics, Suwon, Korea).

\section{Materials}

Phenylephrine, prazosin, phenoxybenzamine, phentolamine, 5-hydroxytryptamine, L-NAME and acetylcholine were obtained from Sigma-Aldrich (St. Louis, Missouri, USA). Dexmedetomidine was donated from Orion Pharma (Turku, Finland). DMEM, FBS, penicillin and streptomycin were obtained from Gibco BRL (Rockville, MD, USA). Phentolamine and phenoxybenzamine were dissolved in ethanol. Unless otherwise stated, we dissolved and diluted all other drugs in distilled water.

\section{Data analysis}

Dexmedetomidine concentration-response curves are expressed as the percentage of maximal contraction induced by phenylephrine or 5-hydroxytryptamine. Concentration-response curves induced by phenylephrine are expressed as the percentage of maximal contraction induced by isotonic $60 \mathrm{mM} \mathrm{KCl}$. The effects of phenylephrine, 5-hydroxytyptamine and endothelial denudation on the dexmedetomidine concentration-response curves and the effects of various drugs on the phenylephrine 
dose-response curves were analyzed through two-way repeated measurement analysis of variance (ANOVA) followed by Bonferroni's post-test. Prazosin concentration-response curves were analyzed using repeated measures ANOVA followed by Bonferroni's post-test. The effects of dexmedetomidine alone or combined treatment with rauwolscine and dexmedetomidine on phenylephrine-induced eNOS phosphorylation in HUVECs were analyzed using one-way ANOVA followed by Bonferroni's post-test. Data are expressed as the mean $\pm \mathrm{SD}$. $\mathrm{N}$ indicates the number of rats from which descending thoracic aortic rings were derived or the number of isolated rat thoracic aortic rings. $P$ values less than 0.05 were considered statistically significant.

\section{Results}

A high dose $\left(10^{-7}\right.$ to $\left.10^{-6} \mathrm{M}\right)$ of dexmedetomidine attenuated phenylephrine-induced contraction (Fig. 1; $P<0.05$ versus $10^{-9} \mathrm{M}$ dexmedetomidine) in endothelium-intact aortae, endothelium-intact aortae pretreated with L-NAME $\left(10^{-4} \quad \mathrm{M}\right)$ and endothelium-denuded aortae pretreated with L-NAME $\left(10^{-4} \mathrm{M}\right)$. However, the dexmedetomidine $\left(10^{-7}\right.$ to $\left.10^{-6} \mathrm{M}\right)$-mediated attenuation of phenylephrine-induced contraction was greater in endothelium-intact aortae than in endothelium-intact or endothelium-denuded aortae pretreated with L-NAME (Fig. 1; $P<0.05$ ). Combined treatment with either dexmedetomidine $\left(3 \times 10^{-7}\right.$ or $\left.10^{-6} \mathrm{M}\right)$ and phenoxybenzamine $\left(5 \times 10^{-8} \mathrm{M}\right)$ or phentolamine $\left(10^{-6}\right.$ $\mathrm{M})$ and phenoxybenzamine $\left(5 \times 10^{-8} \mathrm{M}\right)$ enhanced phenylephrine-induced contraction compared with phenoxybenzamine alone $\left(5 \times 10^{-8} \mathrm{M}\right)$ (Fig. $2 ; P<0.001$ at $10^{-7}$ to $10^{-5} \mathrm{M}$ phenylephrine). In addition, combined treatment with phentolamine and phenoxybenzamine enhanced phenylephrine-induced contraction compared with dexmedetomidine $\left(10^{-6} \mathrm{M}\right)$ and phenoxybenzamine combined treatment (Fig. 2; $P$ $<0.001$ at $10^{-8}$ and $3 \times 10^{-8} \mathrm{M}$ phenylephrine). Combined treatment with high concentrations of dexmedetomidine $\left(10^{-6} \mathrm{M}\right)$ and phenoxybenzamine enhanced phenylephrine-induced contraction compared with combined treatment with low concentrations of dexmedetomidine $\left(3 \times 10^{-7} \mathrm{M}\right)$ and phenoxybenzamine (Fig. 2; $P<0.05$ at $3 \times 10^{-8}$ to $3 \times$ $10^{-7} \mathrm{M}$ phenylephrine). Dexmedetomidine had no effect on 5-hydroxytryptamine-induced contraction in isolated L-NAME-pretreated endothelium-denuded aortae, whereas a high dose $\left(10^{-7}\right.$ to $\left.10^{-6} \mathrm{M}\right)$ of dexmedetomidine attenuated phenylephrine-induced contraction (Fig. 3A; $P<0.001$ versus 5-hydroxytryptamine). Prazosin $\left(3 \times 10^{-9} \mathrm{M}\right)$ attenuated dexmedetomidine-induced contraction (Fig. 3B; $P<0.001$ versus $10^{-6} \mathrm{M}$ dexmedetomidine).

Phenylephrine $\left(10^{-8} \quad \mathrm{M}\right)$ induced eNOS phosphorylation in HUVECs (Fig. $4 ; P<0.001$ versus the control), and dexmedetomidine $\left(3 \times 10^{-7} \mathrm{M}\right)$ enhanced phenylephrine (10-8 M)-induced eNOS phosphorylation (Fig. 4; $P<0.001$ versus phenylephrine alone). Pretreatment with the alpha-2 adrenoceptor antagonist rauwolscine $\left(10^{-6} \mathrm{M}\right)$ attenuated the dexmedetomidine-mediated increase of phenylephrine-induced eNOS phosphorylation (Fig. 4; $P<0.001$ versus dexmedetomidine plus phenylephrine).

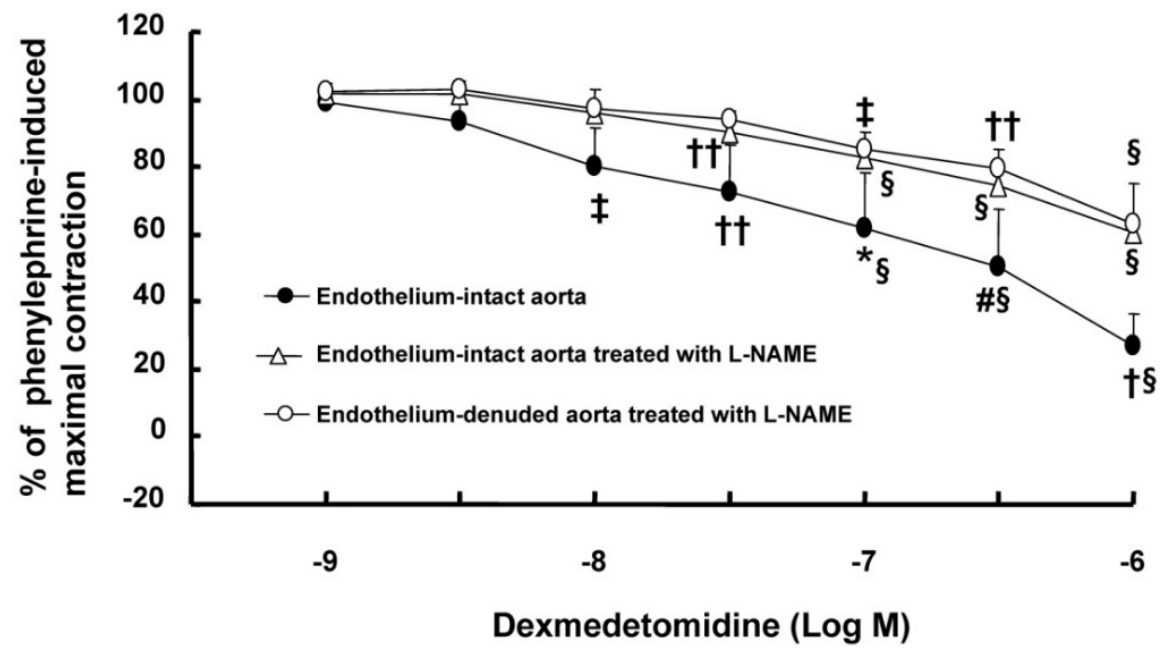

Figure 1. Cumulative dexmedetomidine concentration-response curves for isolated endothelium-intact aortae ( $N=5)$ precontracted with $10-6 \mathrm{M}$ phenylephrine; endothelium-denuded aorta $\left(\mathrm{N}=5\right.$ ) pretreated with $\mathrm{N}^{\omega}$-nitro-L-arginine methyl ester (L-NAME, 10-4 M) and precontracted with 10-7 $M$ phenylephrine; or endothelium-intact aorta $(N=7)$ pretreated with L-NAME $\left(10^{-4} \mathrm{M}\right)$ and precontracted with $10^{-6} \mathrm{M}$ phenylephrine. The data are shown as the mean \pm SD and expressed as the percentage of maximal contraction induced by phenylephrine. $\mathrm{N}$ indicates the number of rats from which descending thoracic aortic rings were derived or the number of isolated rat aortae. ${ }^{*} P<0.05, \# P<0.01$ and $+P<0.001$ versus endothelium-intact and endothelium-denuded aortae treated with L-NAME. $\ddagger P<0.05, \dagger \dagger P<0.01$ and $\S P<0.001$ versus dexmedetomidine $\left(10^{-9} M\right)$ in each group. 


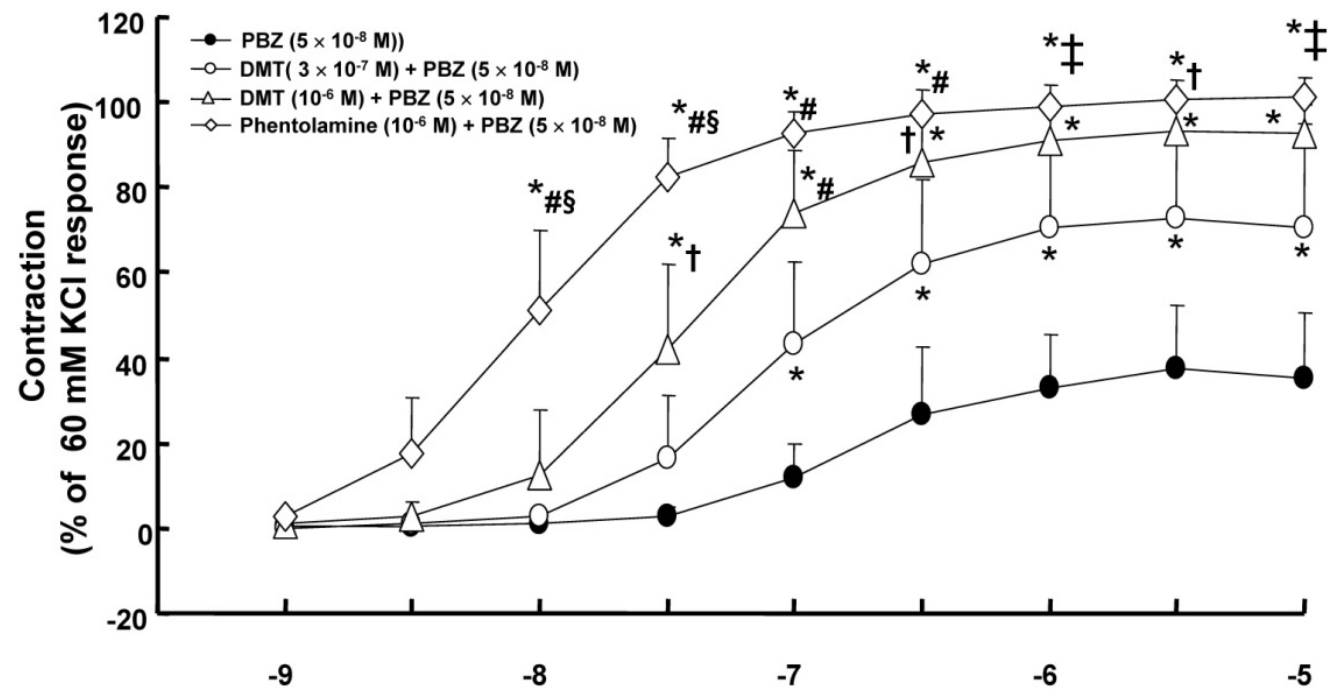

Phenylephrine (Log M)

Figure 2. Effect of combined treatment with either dexmedetomidine (DMT, $3 \times 10^{-7}$ or 10-6 $\mathrm{M} ; \mathrm{N}=6$ ) and phenoxybenzamine (PBZ) or phentolamine ( $N=6$ ) and PBZ, or treatment with PBZ alone $(\mathrm{N}=8)$ on phenylephrine-induced concentration-response curves in isolated endothelium-denuded aortae pretreated with $\mathrm{N}^{\omega}$-nitro-L-arginine methyl ester $\left(10^{-4} \mathrm{M}\right)$. The isolated endothelium-denuded rat aortae were pretreated with DMT or phentolamine, followed by post-treatment with PBZ. Control rings were treated with PBZ alone. Then, all of the aortic rings pretreated with PBZ, DMT and phenoxybenzamine were washed out with fresh Krebs solution. After baseline resting tension had recovered, phenylephrine concentration-response curves were obtained. The data are shown as the mean \pm SD and expressed as the percentage of maximal contraction induced by isotonic $60 \mathrm{mM} \mathrm{KCl}$. N indicates the number of rats from which descending thoracic aortic rings were derived. $* P<0.001$ versus PBZ alone. $\dagger P<0.05, \ddagger P<0.01$ and $\# P<0.001$ versus DMT $\left(3 \times 10^{-7} M\right)+P B Z\left(5 \times 10^{-8} \mathrm{M}\right) . \S P<0.001$ versus DMT $\left(10^{-6} \mathrm{M}\right)+P B Z(5 \times$ 10-8 M).
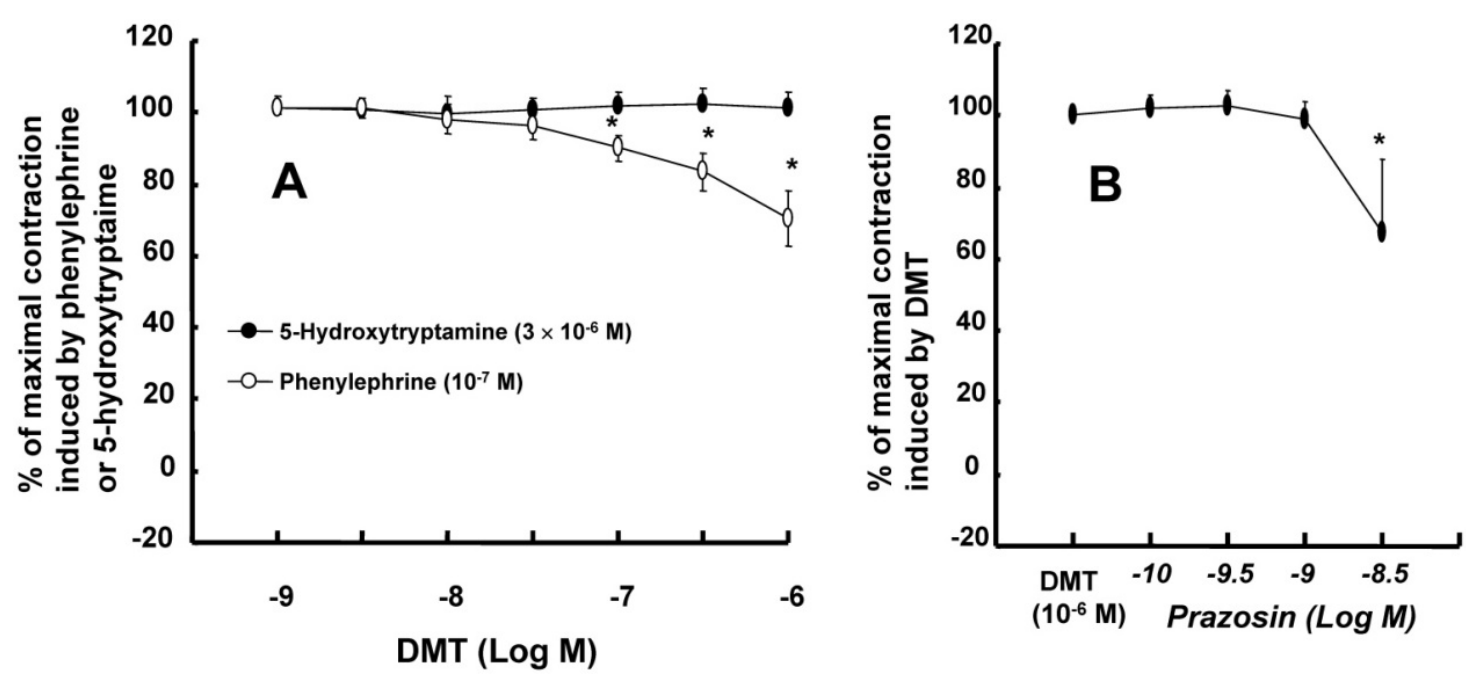

Figure 3. A: Cumulative dexmedetomidine (DMT) concentration-response curves $(N=8)$ for $N^{\omega}$-nitro-L-arginine methyl ester (L-NAME, 10-4 M)-pretreated endothelium-denuded rat aortae precontracted with phenylephrine (10-7 M) or 5-hydroxytryptamine $\left(3 \times 10^{-6} \mathrm{M}\right)$. The data are shown as the mean \pm SD and are expressed as the percentage of maximal contraction induced by phenylephrine or 5 -hydroxytryptamine. $N$ indicates the number of descending thoracic aortic rings. $* P<0.001$ versus 5-hydroxytryptamine. B: Cumulative prazosin concentration-response curves $(N=7)$ for L-NAME $\left(10^{-4} M\right)$-pretreated endothelium-denuded rat aortae precontracted with DMT (10-6 M). The data are shown as the mean \pm SD and are expressed as the percentage of maximal contraction induced by DMT. N indicates the number of descending thoracic aortic rings. $* P<0.001$ versus DMT $\left(10^{-6} \mathrm{M}\right)$.

\section{Discussion}

This is the first study to provide laboratory evidence that dexmedetomidine attenuates alpha-1 adrenoceptor-mediated phenylephrine-induced contraction via blockade of the alpha-1 adrenoceptor. Previous studies have shown that fentanyl attenuates alpha-1 adrenoceptor-mediated phenylephrineinduced vasoconstriction [16,17]. Additionally, dexmedetomidine has been shown to inhibit phenylephrine-induced contraction in the internal mammary and gastroepiploic arteries, suggesting that dexmedetomidine may have an intrinsic affinity for the alpha- 1 adrenoceptor $[7,8]$. Combined treatment with the reversible alpha- 1 adrenoceptor antagonist phentolamine and, subsequently, the irreversible alpha-1 adrenoceptor antagonist phenoxybenzamine enhanced phenylephrine-induced contraction 
compared with pretreatment with phenoxybenzamine alone (Fig. 2), suggesting that the reversible occupation of alpha-1 adrenoceptors in vascular smooth muscle by phentolamine appears to spare them from irreversible blockade by phenoxybenzamine, which contributes to enhanced phenylephrine-induced contraction after washout of all of the pretreatment drugs (phenylephrine, dexmedetomidine and phenoxybenzamine) with Krebs solution. In addition, the supraclinical dose $\left(10^{-7}\right.$ to $\left.10^{-6} \mathrm{M}\right)$ of dexmedetomidine used in the current study, which exceeds the clinically relevant concentration $\left(8 \times 10^{-9} \mathrm{M}\right)$ of dexmedetomidine for sedation, attenuated alpha- 1 adrenoceptor-mediated phenylephrine-induced contraction [18]. Combined pretreatment with dexmedetomidine and phenoxybenzamine enhanced phenylephrine-induced contraction compared with phenoxybenzamine alone (Fig. 2). Taken together, these results suggest that the reversible occupation of alpha-1 adrenoceptors by dexmedetomidine may provide them with partial protection form irreversible blockade by phenoxybenzamine in a dose-dependent manner. The decreasing order of the magnitude of the reversible blockade of alpha-1 adrenoceptors observed in the current study was as follows: phentolamine, $10^{-6} \mathrm{M}$ dexmedetomidine, $3 \times 10^{-7} \mathrm{M}$ dexmedetomidine. Furthermore, dexmedetomidine attenuated phenylephrine-induced contraction, whereas it did not significantly alter contraction induced by the 5-hyroxytryptamine receptor agonist 5-hydroxy- tryptamine, suggesting that dexmedetomidinemediated inhibition appears to be associated with alpha-1 adrenoceptor blockade. In addition, dexmedetomidine-induced contraction is mediated by the alpha-2B and alpha- 1 adrenoceptors in mesenteric arteries [19]. In agreement with a previous report, high-dose dexmedetomidine-induced contraction was attenuated by prazosin at a concentration of $3 \times 10^{-9} \mathrm{M}$, which corresponds to the concentration of prazosin necessary to inhibit alpha-1 adrenoceptors [16,19]. Dexmedetomidine shows a low affinity for the alpha- 1 adrenoceptor and is a partial agonist of the human alpha-1B adrenoceptor $[9,10]$. Taking previous findings into consideration, the putative mechanism associated with the dexmedetomidine-mediated inhibition of phenylephrine-induced contraction is as follows $[9,10]$. Although dexmedetomidine is a highly selective alpha-2 adrenoceptor agonist $\left(\alpha_{2} / \alpha_{1}\right.$ selectivity ratio: 1620) compared with other alpha-2 adrenoceptor agonists, at high doses, dexmedetomidine may occupy a small proportion of alpha-1 adrenoceptors [2]. Thus, because of the competitive inhibition of alpha- 1 adrenoceptors by phenylephrine and high-dose dexmedetomidine, the number of unoccupied alpha-1 adrenoceptors available for phenylephrine binding may decrease, leading to dexmedetomidine-mediated inhibition of phenylephrine-induced contraction.

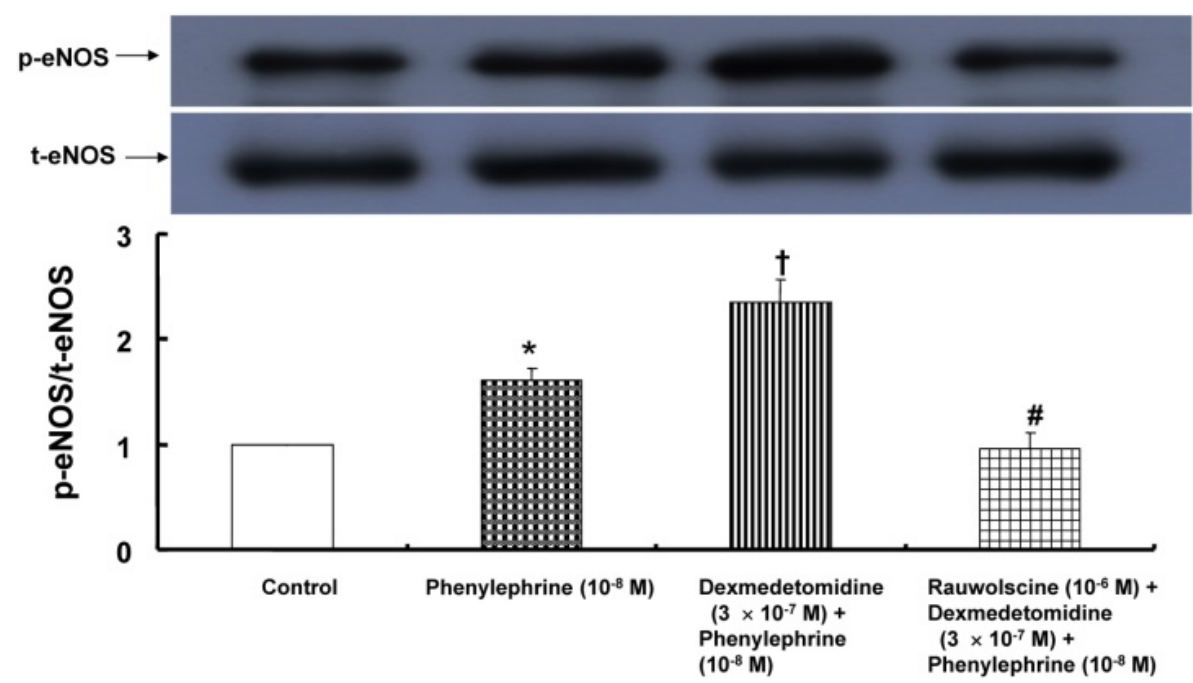

Figure 4. Effect of dexmedetomidine alone $(N=3)$ or combined treatment $(N=3)$ with rauwolscine and dexmedetomidine on phenylephrine-induced endothelial nitric oxide synthase (eNOS) phosphorylation at Serl 177 in human umbilical vein endothelial cells (HUVECs). HUVECs were treated with phenylephrine (10-8 M) alone for $1 \mathrm{~min}$; or pretreated with dexmedetomidine $\left(3 \times 10^{-7} \mathrm{M}\right)$ for $10 \mathrm{~min}$, followed by treatment with 10-8 $\mathrm{M}$ phenylephrine for 1 min; or pretreated with 10-6 $\mathrm{M}$ rauwolscine for $1 \mathrm{~h}$, followed by post-treatment with dexmedetomidine $\left(3 \times 10^{-7} \mathrm{M}\right)$ for 10 min and subsequent treatment with $10^{-8} \mathrm{M}$ phenylephrine for 1 min. The phosphorylation of eNOS was investigated as described in the Methods. The data are shown as the mean \pm SD. N indicates the number of independent experiments. $* P<0.001$ versus the control. $\dagger P<0.001$ versus phenylephrine alone. $\# P<0.001$ versus dexmedetomidine plus phenylephrine. $P$-eNOS: phosphorylated eNOS. t-eNOS: total eNOS. 
Int. J. Med. Sci. 2017, Vol. 14 
Dexmedetomidine-induced contraction is attenuated by endothelial nitric oxide $[11,20]$. Similar to previous reports, the magnitude of the dexmedetomidine-mediated attenuation of phenylephrine-induced contraction was higher in endothelium-intact aortae than in endotheliumdenuded aortae or endothelium-intact aortae pretreated with L-NAME (Fig. 1). However, the dexmedetomidine-mediated inhibition of phenylephrine-induced contraction was not significantly altered in endothelium-denuded aortae or endothelium-intact aortae pretreated with L-NAME (Fig. 1). These results suggest that the dexmedetomidine-mediated attenuation of phenylephrine-induced contraction is partially mediated by endothelial nitric oxide. In agreement with this finding from tension analysis, further experiments showed that rauwolscine attenuated the dexmedetomidine-mediated enhancement of phenylephrine-induced eNOS phosphorylation, suggesting that dexmedetomidine-induced alpha-2 adrenoceptor-mediated nitric oxide attenuates phenylephrine-induced contraction.

Based on these results, when hypotension induced by high-dose dexmedetomidine infusion is encountered, inotropic agents and drugs inhibiting nitric oxide synthesis should be more effective for treating hypotension than alpha-1 adrenoceptor agonists. In the present study, we used isolated the rat aorta, which is regarded as a conduit vessel, whereas the total peripheral vascular resistance associated with blood pressure is determined mainly by small resistance arterioles, such as the mesenteric arteries. Even with this limitation, the dexmedetomidine-mediated inhibition of phenylephrine-induced contraction observed in the current study may contribute to an increased requirement for phenylephrine to treat hypotension induced by dexmedetomidine infusion for sedation and enhanced analgesia [4-6].

In conclusion, our results suggest that dexmedetomidine attenuates phenylephrine-induced contraction via both alpha- 1 adrenoceptor blockade in vascular smooth muscle and enhanced endothelial nitric oxide release in the isolated rat aorta.

\section{Acknowledgements}

This study was supported by a faculty research grant of Yonsei University College of Medicine for (6-2014-0029).

\section{Competing Interests}

The authors have declared that no competing interest exists.

\section{References}

1. Coursin DB, Coursin DB, Maccioli GA. Dexmedetomidine. Curr Opin Crit Care 2001; 7:221-6

2. Virtanen R, Savola JM, Saano V, Nyman L. Characterization of the selectivity, specificity and potency of medetomidine as an alpha 2-adrenoceptor agonist. Eur J Pharmacol 1988; 150:9-14

3. Shirasaka T, Qiu DL, Kannan H, Takasaki M. The effects of centrally administered dexmedetomidine on cardiovascular and sympathetic function in conscious rats. Anesth Analg 2007; 105:1722-8

4. Khalil M, Al-Agaty A, Asaad O, Mahmoud M, Omar AS, Abdelrazik A, et al. A comparative study between propofol and dexmedetomidine as sedative agents during performing transcatheter aortic valve implantation. J Clin Anesth 2016; 32:242-7.

5. Naik BI, Nemergut EC, Kazemi A, Fernández L, Cederholm SK, McMurry TL, et al. The effect of dexmedetomidine on postoperative opioid consumption and pain after major spine surgery. Anesth Analg 2016; 122:1646-53

6. John S, Somal J, Thebo U, Hussain MS, Farag E, Dupler S, et al. Safety and Hemodynamic profile of propofol and dexmedetomidine anesthesia during intra-arterial acute stroke therapy. J Stroke Cerebrovasc Dis 2015; 24:2397-403.

7. Yildiz O, Ulusoy HB, Seyrek M, Gul H, Yildirim V. Dexmedetomidine produces dual alpha2-adrenergic agonist and alpha1-adrenergic antagonist actions on human isolated internal mammary artery. J Cardiothorac Vasc Anesth 2007; 21:696-700.

8. Hamasaki J, Tsuneyoshi I, Katai R, Hidaka T, Boyle WA, Kanmura Y. Dual alpha(2)-adrenergic agonist and alpha(1)-adrenergic antagonist actions of dexmedetomidine on human isolated endothelium-denuded gastroepiploic arteries. Anesth Analg 2002; 94:1434-40.

9. Rayment SJ, Simpson JA, Eames T, Acheson AG, Dashwood MR, Henry Y, et al. Dual effects of a2 -adrenoceptors in modulating myogenic tone in sheep isolated internal anal sphincter. Neurogastroenterol Motil 2014; 26:1095-103.

10. Lehtimäki J, Leino T, Koivisto A, Viitamaa T, Lehtimäki T, Haapalinna A, et al. In vitro and in vivo profiling of fadolmidine, a novel potent alpha(2)-adrenoceptor agonist with local mode of action. Eur J Pharmacol 2008; 599:65-71.

11. Kim HJ, Sohn JT, Jeong YS, Cho MS, Kim HJ, Chang KC, et al. Direct effect of dexmedetomidine on rat isolated aorta involves endothelial nitric oxide synthesis and activation of the lipoxygenase pathway. Clin Exp Pharmacol Physiol 2009; 36:406-12.

12. Ok SH, Kim WH, Yu J, Lee Y, Choi MJ, Lim DH, et al. Effects of acidification and alkalinization on the lipid emulsion-mediated reversal of toxic dose levobupivacaine-induced vasodilation in the isolated rat aorta. Int J Med Sci 2016; 13:68-76.

13. Ok SH, Kwon SC, Kang S, Choi MI, Sohn JT. Mepivacaine-induced intracellular calcium increase appears to be mediated primarily by calcium influx in rat aorta without endothelium. Korean J Anesthesiol 2014; 67:404-11.

14. Ok SH, Bae SI, Kwon SC, Park JC, Kim WC Park KE, et al Bupivacaine-induced vasodilation is mediated by decreased calcium sensitization in isolated endothelium-denuded rat aortas precontracted with phenylephrine. Korean J Pain 2014; 27:229-38.

15. Toda N, Hatano Y. Alpha-adrenergic blocking action of fentanyl on the isolated aorta of the rabbit. Anesthesiology 1977; 46:411-6

16. Sohn JT, Ding X, McCune DF, Perez DM, Murray PA. Fentanyl attenuates alpha1B-adrenoceptor-mediated pulmonary artery contraction. Anesthesiology 2005; 103:327-34.

17. Park KE, Sohn JT, Jeong YS, Sung HJ, Shin IW, Lee HK, et al. Inhibitory effect of fentanyl on phenylephrine-induced contraction of the rat aorta. Yonsei Med J 2009; 50:414-21.

18. Ebert TJ, Hall JE, Barney JA, Uhrich TD, Colinco MD. The effects of increasing plasma concentrations of dexmedetomidine in humans. Anesthesiology 2000; 93:382-94.

19. Wong ES, Man RY, Vanhoutte PM, Ng KF. Dexmedetomidine induces both relaxations and contractions, via different \{alpha\}2-adrenoceptor subtypes, in the isolated mesenteric artery and aorta of the rat. J Pharmacol Exp Ther 2010; 335:659-64.

20. Shafaroudi MM, McBride M, Deighan C, Wokoma A, Macmillan J, Daly CJ, et al. Two "knockout" mouse models demonstrate that aortic vasodilatation is mediated via alpha2a-adrenoceptors located on the endothelium. J Pharmacol Exp Ther 2005; 314:804-10. 